\title{
Evolution of Brain Volume Loss Rates in Early Stages of Multiple Sclerosis
}

Tomas Uher, MD, PhD, Jan Krasensky, MS, Charles Malpas, MPsych, PhD, Niels Bergsland, MS, PhD, Michael G. Dwyer, MS, PhD, Eva Kubala Havrdova, MD, PhD, Manuela Vaneckova, MD, PhD, Dana Horakova, MD, PhD, Robert Zivadinov, MD, PhD, and Tomas Kalincik, MD, PhD

Neurol Neuroimmunol Neuroinflamm 2021;8:e979. doi:10.1212/NXI.0000000000000979

\begin{abstract}
\section{Objective}

To describe the dynamics of brain volume loss (BVL) at different stages of relapsing-remitting multiple sclerosis (RRMS), to describe the association between BVL and clinical measures, and to investigate an effect of treatment escalation on the rate of BVL.
\end{abstract}

\section{Methods}

Together, 1903 patients predominantly with RRMS from the Avonex-Steroids-Azathioprine cohort $(\mathrm{N}=166)$, the study of early IFN- $\beta 1$ la treatment cohort $(\mathrm{N}=180)$, and the quantitative MRI cohort $(\mathrm{N}=1,557)$ with $\geq 2$ MRI scans and $\geq 1$-year of follow-up were included. Brain MRI scans $(N=7,203)$ were performed using a single 1.5-T machine. Relationships between age or disease duration and global and tissue-specific BVL rates were analyzed using mixed models.

\section{Results}

Age was not associated with the rate of $\mathrm{BVL}(\beta=-0.003$; Cohen $\mathrm{f} 2=0.0005$; adjusted $p=0.39)$. Although disease duration was associated with the rate of BVL, its effect on the BVL rate was minimal $\left(\beta=-0.012\right.$; Cohen $\mathrm{f} 2=0.004$; adjusted $\left.p=4 \times 10^{-5}\right)$. Analysis of association between tissue-specific brain volume changes and age $(\beta=-0.019$ to -0.011 ; adjusted $p=0.028-1.00)$ or disease duration $(\beta=-0.028$ to -0.008 ; adjusted $p=0.16-0.96)$ confirmed these results. Although increase in the relapse rate $\left(\beta=0.10\right.$; adjusted $\left.p=9 \times 10^{-9}\right)$, Expanded Disability Status Scale (EDSS; $\beta=0.17$; adjusted $\left.p=8 \times 10^{-5}\right)$, and EDSS change $(\beta=0.15$; adjusted $p=2$ $\times 10^{-5}$ ) were associated with accelerated rate of BVL, their effect on the rate of BVL was minimal (all Cohen $\mathrm{f} 2 \leq 0.007$ ). In 94 patients who escalated therapy, the rate of BVL decreased following treatment escalation by $0.29 \%\left(\beta=-0.29\right.$; Cohen $\left.\mathrm{f} 2=0.133 ; p=5.5 \times 10^{-8}\right)$.

\section{Conclusions}

The rate of BVL is relatively stable throughout the course of RRMS. The accelerated BVL is weakly associated with concurrent higher disease activity, and timely escalation to high-efficacy immunotherapy helps decrease the rate of BVL.

\author{
Correspondence \\ Dr. Uher \\ tomas.uher@vfn.cz
}




\section{Glossary}

ASA = Avonex-Steroids-Azathioprine; $\mathbf{B V L}=$ brain volume loss; $\mathbf{C I S}=$ clinically isolated syndrome; $\mathbf{D M T}=$ disease-modifying treatment; EDSS = Expanded Disability Status Scale; MS = multiple sclerosis; QMRI = Quantitative MRI; RRMS = relapsingremitting MS; SET = Study of Early IFN- $\beta 1$ a Treatment; SPMS = secondary progressive MS.

Accelerated whole-brain volume loss (BVL) in patients with multiple sclerosis (MS) is associated with an increased risk of neurologic and cognitive impairment. Assessment of BVL may help to evaluate early treatment response and improve the stratification of MS patients who are at highest risk of disease progression. ${ }^{1-5}$ Although BVL is clinically relevant, our understanding of BVL dynamics throughout the disease course is limited. Previous studies have reported contradictory findings. Although some studies have suggested constant BVL rate throughout the MS disease course, ${ }^{6,7}$ other studies have reported accelerated BVL rate in early ${ }^{8}$ or advanced ${ }^{2,9}$ disease stages.

It is widely accepted that patients reaching secondary progressive phase have in general greater disease severity than age-matched patients with clinically isolated syndrome (CIS) or relapsing-remitting MS (RRMS). However, the previous studies investigating BVL dynamics in MS compared BVL rates between CIS or RRMS and secondary progressive MS (SPMS). Comparison of patients with different MS phenotypes but also with different disease severity may introduce bias into longitudinal evaluation of BVL trajectory. Only 1 study examined evolution of BVL rate on a continuous scale, but was limited by having a relatively small number of time points over a limited follow-up duration. ${ }^{8}$

A thorough understanding of the average BVL trajectory throughout the course of MS is important for balanced recruitment of patients into clinical studies where BVL is used as an outcome measure. Furthermore, an expectation of higher rate of BVL in certain stages of MS would increase clinical awareness during such periods and might lead to timely intensified therapeutic intervention in patients exhibiting higher disease activity, on the proviso that such therapies can mitigate the rate of BVL. Given that the frequency of relapses is highest at young age and during early disease, ${ }^{10-12}$ and that highly inflammatory MS phenotype is associated with faster BVL, ${ }^{13}$ we hypothesized that BVL is accelerated in early stages of MS. In this study, we investigated the dynamics of BVL and tissue-specific brain volume changes in patient with different ages and disease durations. Given that previous studies showed a spatiotemporal pattern of gray and white matter volume changes during aging ${ }^{14,15}$ and MS progression, ${ }^{2,8,14}$ we also investigated an evolution of tissue-specific brain volume changes through disease course, such as total, cortical and deep gray matter, white matter, thalamus, and lateral ventricle. The secondary aims were to describe association between BVL and clinical measures and to investigate an effect of treatment change on the rate of BVL.

\section{Methods}

\section{Patients}

We analyzed a combined cohort of adult (aged $>18$ years), predominantly patients with RRMS, who participated in the Avonex-Steroids-Azathioprine (ASA) trial $(\mathrm{N}=166)$, Study of Early IFN- $\beta 1$ 1a Treatment (SET) $(\mathrm{N}=180)$, and in a cohort of patients from the quantitative MRI (QMRI) project $(\mathrm{N}=1557)$.

\section{ASA Cohort}

The original ASA study was a 2-year randomized, double-blind, placebo-controlled trial with a 5-year open-label extension, investigating clinical and MRI outcomes among patients with RRMS treated with IFNß-1a (enrollment from April 1999 to December 2003). ${ }^{16}$

\section{SET Cohort}

The original SET study was a 2-year investigator initiated, prospective, observational clinical study of IFNB-1a treatment in patients after the first demyelinating event suggestive of MS involving 8 centers (enrollment from October 2005 to June 2009; clin.gov \#NCT01592474). ${ }^{17}$

\section{QMRI Cohort}

All patients with MS routinely followed at the MS Centre at the General University Hospital in Prague, and with brain MRI from March 2000 to November 2015, were included in the QMRI program. ${ }^{18}$

\section{Clinical Evaluation}

After the SET and ASA were completed, patients' standard clinical follow-up, consisting of 3- to 6-monthly visits, was aligned with the QMRI cohort. In this study, we included clinical data collected before November 2015. Confirmed disability worsening was defined as worsening by an increase in the Expanded Disability Status Scale (EDSS) of 1.5 points if the baseline EDSS score was 0; 1.0 point if the baseline EDSS score was between 1.0 and 5.0; and 0.5 point for baseline EDSS scores of 5.5 or higher. We required confirmation of disability worsening after 12 months. ${ }^{19}$ Additional details about study cohorts and clinical evaluations are provided in supplemental material 1, links.lww.com/NXI/A448.

\section{Selection Criteria}

We excluded MRI scans performed: within 1 month after clinical relapse or treatment with high-dose steroids, within 10 months (MRI monitoring every $12 \pm 2$ months) after previous MRI scan, in pregnancy, or within 12 months after delivery. In addition, MRI scans performed within first 12 months after diseasemodifying treatment (DMT) initiation or DMT escalation were excluded to prevent confounding effect of pseudo-atrophy. ${ }^{20,21}$ 
DMT escalation was defined as switching from low-efficacy DMT (dimethyl fumarate, glatiramer acetate, interferon $\beta$, and teriflunomide) to high/moderate-efficacy DMT (fingolimod, mitoxantrone, and natalizumab). Finally, we included only patients with $\geq 1$-year ( \pm 2 months) follow-up and $\geq 2$ MRI scans.

\section{Ethics Statement}

The study protocols were approved by the medical ethics committees, and all patients gave their written informed consent.

\section{MRI Acquisition and Analysis}

All MRI scans analyzed in the study were performed on the same scanner (1.5-T Gyroscan; Philips Medical Systems, Best, the Netherlands) that did not undergo a major hardware upgrade. The standardized protocol consisted of 2 sequences: fluidattenuated inversion recovery and $\mathrm{T} 1$-weighted 3-dimensional turbo field echo (T1-WI/TFE 3D). Volumetric assessment of T2 lesions and brain volumes was performed using semiautomated ScanView software. Lesion filling on T1-WI 3D images was used to reduce the impact of $\mathrm{T} 1$ hypointensities on tissue segmentation. ${ }^{22}$ For longitudinal changes of the BVL, we applied the SIENA technique. ${ }^{23}$ In MRI scans from the ASA and SET cohorts, we performed validation analysis of tissue-specific brain volumes by FMRIB's Integrated Registration and Segmentation Tool $^{24}$ and SIENAX multi-time-point. ${ }^{25}$ Details of MRI acquisition and analysis are described in supplemental material 2, links.lww.com/NXI/A449.

\section{Statistical Analysis}

All analyses were performed using the $\mathrm{R}$ statistical software (R-project.org). We analyzed annualized \% volume changes (BVL rate) relative to the preceding MRI examination.

\section{The Relationship Between Age or Disease Duration as Continuous Variables and BVL Rate} The relationship between age or disease duration (independent variables) and annualized BVL rate (dependent variable) was analyzed using linear mixed models with a random intercept specified for each patient. We estimated effect size (Cohen $\mathrm{f} 2$ ) for the effect of age and disease duration on the BVL rate. Cohen $\mathrm{f} 2 \geq 0.02, \mathrm{f} 2 \geq 0.15$, and $\mathrm{f} 2 \geq 0.35$ represent small, medium, and large effect sizes, respectively. ${ }^{26}$ Regression coefficients $(\beta)$ for the age or disease duration of the models were interpreted as average change of the BVL rate for each 1-year increase in patient age or disease duration.

Mixed models with an interaction term were used to investigate additive effect of age at time point and disease duration at baseline (or vice versa) on evolution of MRI measures. Variance inflation factors were used to examine multicollinearity among independent variables.

\section{The Relationship Between Age or Disease} Duration as Categorical Variables and BVL Rate For each patient, we calculated the mean annual BVL rate at different available ages and disease durations (figure 1) and compared them by mixed models. For each patient, we also fitted individual slopes (e.g., calculated regression coefficients) of the BVL rates at different available age and disease duration intervals using linear regression. These fitted individual BVL slopes (e.g., regression coefficients) were compared by mixed models to investigate differences in BVL trajectories throughout disease course.

\section{The Relationship Between Clinical Disease Activity and the Rate of BVL}

We then analyzed the relationships of annualized relapse rate, EDSS score, and annualized EDSS change (all independent $\log +1$ transformed variables) with BVL rates using mixed models. Mixed models were also used to investigate interactions between age (or disease duration) and measures of clinical disease activity and their associations with MRI measures.

In addition, we analyzed associations between the BVL during the initial 2 years of follow-up and time to confirmed disability worsening using adjusted Cox proportional hazard models. The cutoff point for BVL rate was based on the third quartile. Association between annualized EDSS change over the entire follow-up and age or disease duration at baseline was analyzed by multivariable linear regression.

\section{The Effect of Treatment Change on BVL Rate}

Finally, in a subgroup of patients who had completed at least 2 MRI scans before and 2 MRI scans after DMT escalation, we compared BVL rates before and after DMT escalation using mixed models.

\section{Sensitivity Analyses}

Sensitivity analyses were completed (a) in a subgroup of patients treated with only low-efficacy DMTs to eliminate the effect of treatment change and (b) in trial patients from the ASA and the SET cohorts to analyze association between age and disease duration with tissue-specific brain \% volume changes.

\section{Adjustment of Multivariable Models}

Unless otherwise indicated, multivariable mixed and Cox proportional hazard models were adjusted for sex, study cohort, T2 lesion volume at time point, normalized brain volume (brain parenchymal fraction) at baseline, proportion of time spent on low-efficacy DMTs, and proportion of time spent on high/moderate-efficacy DMTs.

\section{False Discovery Rate Control}

The Holm procedure with $\mathrm{q}=0.05$ was used to control false discovery rate of dependent tests. The number in [brackets] following adjusted $p$ refers to the number of comparisons.

\section{Data Availability}

Anonymized data not published within this article will be made available by reasonable request from any qualified investigator for purposes of replicating study results. 

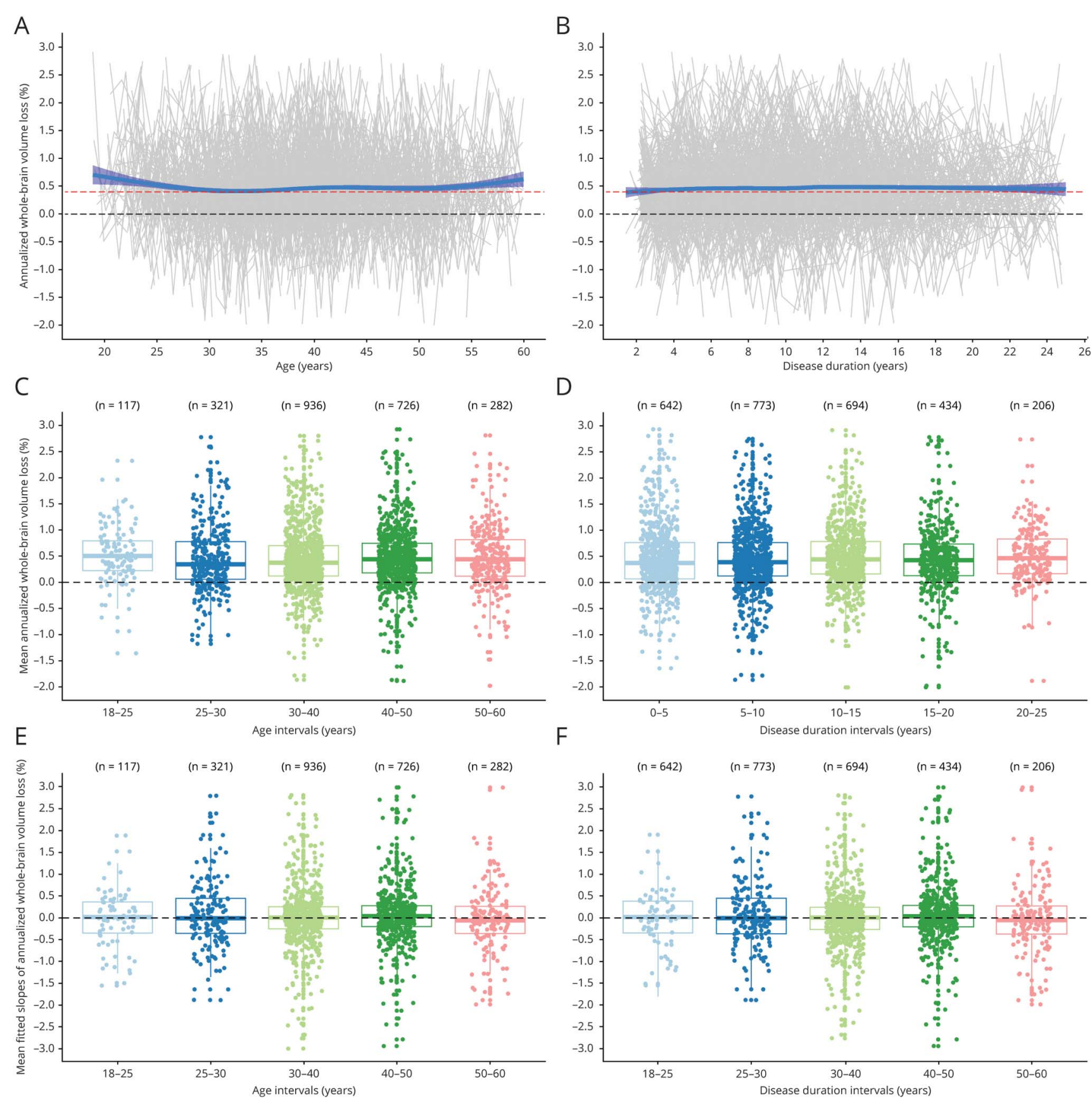

Relationship between annualized whole-brain volume loss rate and age (A) and disease duration as continuous variables (B) with a smooth curve fitted by loess regression technique; relationship between mean annualized whole-brain volume loss rates at different ages (C) and disease duration intervals (D); and relationship between mean fitted individual slopes (regression coefficients) of annualized whole-brain volume loss rate trajectories at different ages (E) and disease duration intervals (F). The dotted black horizontal line represents no change in annualized whole-brain volume loss rate, and the red dotted horizontal line represents the reference cutoff value $(0.4 \%$ per year) for pathologic whole-brain volume change.

\section{Results}

\section{Baseline and Follow-up Clinical Characteristics} Of the 3,415 patients (20,101 MRI scans), 1,903 patients (7,203 MRI scans) fulfilled the inclusion criteria and were included in this study. Age, disease duration, and EDSS at baseline differed among the cohorts (small to median effect sizes). The mean rate of BVL was $0.48 \% \pm 0.93 \%$ among all patients; $0.45 \% \pm 0.94 \%$ among patients without DMTs; $0.50 \% \pm 0.94 \%$ among patients treated with low-efficacy DMTs; and $0.43 \% \pm 0.82 \%$ among patients treated with high/moderate-efficacy DMTs. Table 1 describes the demographic, clinical, and MRI characteristics of the sample.

\section{Brain Volume Loss and Age}

When we explored age as continuous variable, we did not find a significant association between younger age and higher BVL rate 
Table 1 Sample Characteristics

\begin{tabular}{|c|c|c|c|c|c|}
\hline \multirow[b]{2}{*}{ Characteristics } & \multirow[b]{2}{*}{ All } & \multicolumn{3}{|l|}{ Study cohort } & \multirow{2}{*}{$\begin{array}{l}\text { Between-group } \\
\text { comparison } \\
\text { (Eta-squared) }\end{array}$} \\
\hline & & ASA & SET & QMRI & \\
\hline No. of patients & 1,903 & 166 & 180 & 1,557 & \\
\hline Females & $1,341(70.5 \%)$ & $125(77.2 \%)$ & $115(63.9 \%)$ & $1,095(70.6 \%)$ & 0.004 \\
\hline Age at baseline $(y)^{a}$ & $36.2 \pm 8.9$ & $32.2 \pm 7.6$ & $31.2 \pm 7.6$ & $37.3 \pm 8.8$ & $0.059 *$ \\
\hline Disease duration at baseline $(y)^{a}$ & $7.4 \pm 5.7$ & $6.3 \pm 4.3$ & $1.6 \pm 1.1$ & $8.2 \pm 5.7$ & $0.14 * *$ \\
\hline EDSS at baseline ${ }^{b}$ & $2.0(1.0-3.0)$ & $2.0(1.5-3.5)$ & $1.5(1.0-2.0)$ & $2.0(1.5-3.5)$ & $0.056^{*}$ \\
\hline Relapsing-remitting MS (McDonald 2017 criteria) & $1782(93.6 \%)$ & $166(100 \%)$ & $180(100.0 \%)$ & $1,436(92.2 \%)$ & $0.012^{*}$ \\
\hline $\begin{array}{l}\text { Clinically isolated syndrome (McDonald } 2005 \\
\text { criteria) at baseline }\end{array}$ & $642(33.7 \%)$ & $0(0.0 \%)$ & $127(70.6 \%)$ & 549 (35.3\%) & $0.088^{*}$ \\
\hline Total no. of MRI scans & 7,204 & 938 & 606 & 5,651 & - \\
\hline No. of MRI scans per patient ${ }^{b}$ & $4(3-6)$ & $6(4-7)$ & $3(3-4)$ & $3(2-5)$ & $0.11 * *$ \\
\hline Follow-up duration $(y)^{b}$ & $3.4(2.1-5.3)$ & $8.8(7.1-9.0)$ & $3.0(2.0-3.0)$ & $3.4(2.0-5.1)$ & $0.14 * *$ \\
\hline \multicolumn{6}{|l|}{ Treatment at baseline } \\
\hline No DMT & $502(26.4 \%)$ & $5(3.1 \%)$ & $3(1.7 \%)$ & $494(31.7 \%)$ & $0.038 *$ \\
\hline Low-efficacy DMT ${ }^{c}$ & $1,311(68.9 \%)$ & $157(96.9 \%)$ & $175(97.2 \%)$ & $973(62.5 \%)$ & \\
\hline High/moderate-efficacy DMTc & $90(4.7 \%)$ & $0(0.0 \%)$ & $2(1.1 \%)$ & $88(5.7 \%)$ & \\
\hline \multicolumn{6}{|l|}{ Treatment at last visit } \\
\hline No DMT & $521(27.4 \%)$ & $20(12.0 \%)$ & $11(6.1 \%)$ & $490(31.5 \%)$ & \multirow{3}{*}{0.008} \\
\hline Low-efficacy DMTc & $1,117(58.7 \%)$ & $135(81.3 \%)$ & $162(90.0 \%)$ & $820(52.7 \%)$ & \\
\hline High/moderate-efficacy DMT ${ }^{c}$ & $265(13.9 \%)$ & $11(6.6 \%)$ & $7(3.9 \%)$ & $247(15.9 \%)$ & \\
\hline \multicolumn{6}{|l|}{ MRI measures over follow-up } \\
\hline $\begin{array}{l}\text { Average annualized T2 lesion volume absolute } \\
\text { change }^{a}\end{array}$ & $0.25 \pm 1.05$ & $0.37 \pm 1.11$ & $0.12 \pm 0.89$ & $0.24 \pm 1.05$ & 0.004 \\
\hline Average annualized BVL rate ${ }^{a}$ & $-0.48 \pm 0.93$ & $-0.60 \pm 1.33$ & $-0.47 \pm 0.73$ & $-0.46 \pm 0.85$ & 0.003 \\
\hline \multicolumn{6}{|c|}{$\begin{array}{l}\text { Abbreviations: ASA = Avonex-Steroids-Azathioprine; BVL = annualized whole-brain volume loss rate; DMT = disease-modifying treatment; EDSS = Expandec } \\
\text { Disability Status Scale; QMRI = quantitative MRl; SET = study of early IFN- } \beta 1 \text { a treatment. } \\
\text { Effect size statistic for the Kruskal-Wallis test (interpretation of eta-squared: } 0.01=\text { small*, } 0.06=\text { medium**; }>0.14=\text { large effect size }{ }^{\star \star *} \text { ). } \\
\text { a Mean and SD. } \\
\text { b Median and interquartile range. } \\
\text { ' Low-efficacy DMT: glatiramer acetate, dimethyl fumarate, and interferons; high/moderate-efficacy DMT: fingolimod, mitoxantrone, and natalizumab. }\end{array}$} \\
\hline
\end{tabular}

$(\beta=-0.003 ; 95 \% \mathrm{CI}=-0.006$ to 0.0002 ; Cohen $\mathrm{f} 2=0.0005 ; p=$ 0.065 ; adjusted $p[7]=0.39$ ) (table 2 and figure $1 \mathrm{~A}$ ). Similar results were seen when different DMTs groups were analyzed separately (table 2). To eliminate an effect of disease duration on this association, we analyzed patients with disease duration at baseline, of 1,2, or 3 years, but again we did not find an effect of age on BVL rate (all Cohen $\mathrm{f} 2<0.001$ ) (table 2).

When age was analyzed as categorical variable, we found similar BVL rates in different age groups (table 3).

We did not find differences in means of linear regression coefficients of BVL rates among age intervals $(\beta=0.047 ; 95 \%$ $\mathrm{CI}=-0.06$ to 0.15 ; Cohen $\mathrm{f} 2<0.001 ; p=0.40$ ) (figure $1 \mathrm{E}$ ).
Brain Volume Loss and Disease Duration

Although BVL rate tended to decrease with disease duration, the effect of disease duration on BVL rate was minimal $(\beta=$ $-0.012 ; 95 \% \mathrm{CI}=-0.017$ to -0.007 ; Cohen $\mathrm{f} 2=0.004 ; p=6 \times$ $10^{-6}$; adjusted $p[7]=4 \times 10^{-5}$ ) (table 2 and figure $\left.1 \mathrm{~B}\right)$. Among patients on low-efficacy DMTs, each year of disease duration was associated with the mean reduction of 0.009 BVL rate $(\beta=-0.009 ; 95 \% \mathrm{CI}=-0.016$ to -0.003 ; Cohen $\mathrm{f} 2$ $=0.002 ; p=0.005$; adjusted $p[7]=0.024)$. To eliminate $\mathrm{a}$ potential confounding effect of age on this association, we separately analyzed patients with baseline age of 25-30 years, $30-35$ years, or $35-40$ years, but we again did not find any effect of disease duration on BVL rate (all Cohen $\mathrm{f} 2<0.007$ ) (table 2). 
Table 2 Association Between Age (or Disease Duration) and Rates of Whole-Brain Volume Loss in Subgroups of Patients Defined by Their Age/Disease Duration at Baseline or by Their DMT

\begin{tabular}{|c|c|c|c|c|c|}
\hline & Definition of patients included & $\begin{array}{l}\text { N (patients; } \\
\text { MRI scans) }\end{array}$ & $\beta(95 \% \mathrm{Cl})$ & $\begin{array}{l}\text { Cohen } \\
\text { f } 2^{\text {b }}\end{array}$ & $\begin{array}{l}p \text { Value; adjusted } \\
p \text { value }\end{array}$ \\
\hline \multirow{7}{*}{$\begin{array}{l}\text { Association between age and the BVL } \\
\text { rate }\end{array}$} & All patients/MRI scans & 1,$903 ; 7,203$ & $\begin{array}{l}-0.003(-0.006 \\
0.0002)\end{array}$ & 0.001 & $0.065 ; 0.39$ \\
\hline & $\begin{array}{l}\text { Patients with disease duration at } \\
\text { baseline }<1 \mathrm{y}\end{array}$ & $468 ; 1,536$ & $\begin{array}{l}-0.0004 \\
(-0.007,0.006)\end{array}$ & $<0.001$ & $0.91 ; 1.00$ \\
\hline & $\begin{array}{l}\text { Patients with disease duration at } \\
\text { baseline }<2 \text { y }\end{array}$ & $629 ; 2,134$ & $\begin{array}{l}-0.001(-0.007 \\
0.004)\end{array}$ & $<0.001$ & $0.67 ; 1.00$ \\
\hline & $\begin{array}{l}\text { Patients with disease duration at } \\
\text { baseline }<3 y\end{array}$ & $755 ; 2,654$ & $\begin{array}{l}-0.0003 \\
(-0.006,0.005)\end{array}$ & $<0.001$ & $0.91 ; 1.00$ \\
\hline & Only time points without DMTsa & $595 ; 1,786$ & $\begin{array}{l}-0.009(-0.015 \\
-0.003)\end{array}$ & 0.006 & $0.006 ; 0.042^{c}$ \\
\hline & $\begin{array}{l}\text { Only time points during low-efficacy } \\
\text { DMTs }^{a}\end{array}$ & 1,$268 ; 4,817$ & $\begin{array}{l}-0.001(-0.005 \\
0.003)\end{array}$ & $<0.001$ & $0.59 ; 1.00$ \\
\hline & $\begin{array}{l}\text { Only time points during high/ } \\
\text { moderate-efficacy DMTs }\end{array}$ & $272 ; 756$ & $\begin{array}{l}-0.001(-0.011 \\
0.009)\end{array}$ & $<0.001$ & $0.84 ; 1.00$ \\
\hline \multirow{7}{*}{$\begin{array}{l}\text { Association between disease } \\
\text { duration and the BVL rate }\end{array}$} & All patients/MRI scans & 1,$903 ; 7,203$ & $\begin{array}{l}-0.012(-0.017 \\
-0.007)\end{array}$ & 0.004 & $6 \times 10^{-6} ; 4 \times 10^{-5 c}$ \\
\hline & $\begin{array}{l}\text { Patients with age at baseline between } \\
25 \text { and } 30 y\end{array}$ & $331 ; 1,196$ & $\begin{array}{l}0.003(-0.010 \\
0.020)\end{array}$ & $<0.001$ & $0.75 ; 0.75$ \\
\hline & $\begin{array}{l}\text { Patients with age at baseline between } \\
30 \text { and } 35 \text { y }\end{array}$ & $423 ; 1,658$ & $\begin{array}{l}-0.009(-0.020 \\
0.002)\end{array}$ & 0.001 & $0.096 ; 0.19$ \\
\hline & $\begin{array}{l}\text { Patients with age at baseline between } \\
35 \text { and } 40 y\end{array}$ & $359 ; 1,405$ & $\begin{array}{l}-0.020(-0.030 \\
-0.005)\end{array}$ & 0.007 & $0.004 ; 0.024^{c}$ \\
\hline & Only time points without DMTs ${ }^{a}$ & $595 ; 1,786$ & $\begin{array}{l}-0.014(-0.024 \\
-0.004)\end{array}$ & 0.006 & $0.004 ; 0.024^{c}$ \\
\hline & $\begin{array}{l}\text { Only time points during low-efficacy } \\
\text { DMTs }^{a}\end{array}$ & 1,$268 ; 4,817$ & $\begin{array}{l}-0.009(-0.016 \\
-0.003)\end{array}$ & 0.002 & $0.005 ; 0.024^{c}$ \\
\hline & $\begin{array}{l}\text { Only time points during high/ } \\
\text { moderate-efficacy DMTs }\end{array}$ & $272 ; 756$ & $\begin{array}{l}-0.017(-0.032 \\
-0.003)\end{array}$ & 0.006 & $0.020 ; 0.060$ \\
\hline
\end{tabular}

Abbreviations: adjusted $p=$ adjusted $p$ value by Holm procedure to control FDR; $A S A=A$ vonex-Steroids-Azathioprine; BVL = annualized whole-brain volume loss rate; DMT = disease-modifying treatment; FDR = false discovery rate; $\mathrm{N}=$ number; $\mathrm{QMRI}=$ quantitative MRI; SET = study of early IFN- $\beta 1$ a treatment. The relationships between rates of BVL and age or disease duration were analyzed using linear mixed models with a random intercept specified for each subject. Models were adjusted for sex, log+1 transformed T2 lesion volume at time point, normalized brain volume at baseline, study cohort (ASA/QMRI/SET), proportion of time spent on low-efficacy DMTs (dimethyl fumarate, glatiramer acetate, interferon $\beta$, and teriflunomide), and high/moderate-efficacy DMTs (fingolimod, mitoxantrone, and natalizumab) at time point.

a Low-efficacy DMTs: dimethyl fumarate, glatiramer acetate, interferon $\beta$, and teriflunomide; high/moderate-efficacy DMTs (fingolimod, mitoxantrone, and natalizumab).

${ }^{\text {b }}$ Standardized measure of effect size (small effect size: $f 2 \geq 0.02$, medium effect size: $f 2 \geq 0.15$, and large effect size: $f 2 \geq 0.35$ ).

c Statistically significant after correction for false discovery rate using the Holm procedure with $q$ value $<0.05$ and number of comparisons [7].

When age analyzed as categorical variable, we found similar BVL rates across different disease duration intervals (table 3).

We did not find differences in means of linear regression coefficients of the BVL rates among disease duration interval subgroups $(\beta=0.013 ; 95 \% \mathrm{CI}=-0.03$ to 0.06 ; Cohen $\mathrm{f} 2<$ $0.001 ; p=0.59)$ (figure $1 \mathrm{~F})$.

We did not find differential effect of disease duration across different ages on BVL rate (interaction between age at time point and disease duration at baseline: $\beta=-0.00002 ; p=0.41$; adjusted $p$ [2] $=0.41$; interaction between age at baseline and disease duration at time point: $\beta=-0.000001 ; p=0.16$; adjusted $p[2]=0.32$ ).

\section{Association Between Brain Volume Loss and Relapse Activity}

The relapse rate (e.g., number of relapses per year) declined with increasing age and disease duration of the studied patients (figure e-1, links.lww.com/NXI/A443). Increase in relapse rate by 1.0 was associated with the mean BVL rate increase by $0.10 \%(\beta=0.10 ; 95 \% \mathrm{CI}=0.07$ to 0.13 ; Cohen $\mathrm{f} 2$ $=0.007 ; p=3 \times 10^{-9}$; adjusted $p[3]=9 \times 10^{-9}$ ) (figure e-2, links.lww.com/NXI/A444). Younger age was associated with a stronger association between relapse rate and BVL rate (interaction between age and relapse rate: $\beta=0.012 ; p=$ 0.008 ; adjusted $p[3]=0.016$ ). Disease duration did not further modify the association between relapse rate and BVL rate 
Table 3 Rates of Whole-Brain Volume Loss in Subgroups of Patients Defined by Their Age and Disease Duration

\begin{tabular}{|c|c|c|c|c|c|}
\hline & \multirow{2}{*}{$\begin{array}{l}\text { Ranges } \\
\text { (y) }\end{array}$} & \multicolumn{2}{|c|}{ Time between 2 consecutive MRI scans $>10$ mo } & \multicolumn{2}{|c|}{ Time between 2 consecutive MRI scans $>20$ mo } \\
\hline & & $\mathbf{N}$ & Median, mean $(95 \% \mathrm{Cl})$ of BVL within age group & $\mathbf{N}$ & Median, mean $(95 \% \mathrm{CI})$ of $\mathrm{BVL}$ within age group \\
\hline \multirow{5}{*}{ Age } & $18-25$ & 117 & $0.51 ; 0.54(0.44,0.64)$ & 72 & $0.46 ; 0.56(0.43,0.69)$ \\
\hline & $25-30$ & 321 & $0.35 ; 0.45(0.37,0.53)$ & 206 & $0.36 ; 0.43(0.35,0.51)$ \\
\hline & $30-40$ & 936 & $0.39 ; 0.47(0.43,0.51)$ & 683 & $0.40 ; 0.47(0.43,0.51)$ \\
\hline & $40-50$ & 726 & $0.45 ; 0.50(0.45,0.54)$ & 537 & $0.48 ; 0.54(0.50,0.58)$ \\
\hline & $50-60$ & 282 & $0.44 ; 0.50(0.43,0.58)$ & 202 & $0.45 ; 0.46(0.39,0.53)$ \\
\hline \multirow{5}{*}{$\begin{array}{l}\text { Disease } \\
\text { duration }\end{array}$} & $0-5$ & 624 & $0.38 ; 0.49(0.43,0.54)$ & 388 & $0.41 ; 0.44(0.39,0.50)$ \\
\hline & $5-10$ & 773 & $0.40 ; 0.46(0.42,0.51)$ & 549 & $0.40 ; 0.48(0.44,0.53)$ \\
\hline & $10-15$ & 694 & $0.45 ; 0.51(0.46,0.56)$ & 491 & $0.48 ; 0.54(0.48,0.60)$ \\
\hline & $15-20$ & 434 & $0.44 ; 0.47(0.40,0.53)$ & 311 & $0.42 ; 0.46(0.40,0.52)$ \\
\hline & $20-25$ & 206 & $0.47 ; 0.51(0.44,0.59)$ & 154 & $0.46 ; 0.54(0.47,0.62)$ \\
\hline
\end{tabular}

Abbreviation: BVL = annualized whole-brain volume loss.

(interaction between disease duration and relapse rate: $\beta=$ 0.003; $p=0.61$; adjusted $p[3]=0.61$ ).

\section{Association Between Brain Volume Loss and Disability}

The BVL rate was associated with the EDSS score $(\beta=0.17$; $95 \% \mathrm{CI}=0.09$ to 0.24 ; Cohen $\mathrm{f} 2=0.003 ; p=2 \times 10^{-5}$; adjusted $\left.p[5]=8 \times 10^{-5}\right)$ and annualized EDSS absolute change $(\beta=0.15 ; 95 \% \mathrm{CI}=0.09-0.21$; Cohen $\mathrm{f} 2=0.006 ; p=$ $3 \times 10^{-6}$; adjusted $\left.p[5]=2 \times 10^{-5}\right)$. The association between BVL rate and annualized EDSS absolute change was stronger among older patients (interaction between age and BVL rate: $\beta=0.003 ; p=0.00015$; adjusted $p[5]=0.0005$ ). Disease duration did not further modify the association between BVL rate and annualized EDSS absolute change (interaction between disease duration and BVL rate: $\beta=0.001 ; p=0.28$; adjusted $p[5]=0.28$ ).

The mean BVL rate during the first 2 years of the follow-up (cutoff $<-0.87 \%$; third quartile) was associated with a higher risk of confirmed disability worsening over the long term (OR $=1.4 ; 95 \% \mathrm{CI}=1.1-1.7 ; p=0.005$; adjusted $p[5]=0.010$ ) (figure e-3, links.lww.com/NXI/A445). Annualized absolute change in EDSS did not differ among patients with different ages and disease durations (both $p=0.38-0.41$ ) (e-figure 4, links.lww.com/NXI/A446).

\section{Association Between Brain Volume Loss and Lesion Burden}

An average increase in T2 lesion volume was $6.5 \%$ per year (95\% CI = 6.0-7.1; $p<1 \times 10^{-10}$; adjusted $p[8]<1 \times 10^{-10}$ ). We found a weak association between the higher rate of $\mathrm{T} 2$ lesion volume accumulation $(\mathrm{mL})$ and younger age or shorter disease duration. On average, the rate of $\mathrm{T} 2$ lesion volume increase decelerated by $0.006 \mathrm{~mL}$ for every year of age $(\beta=$
$-0.006 ; 95 \% \mathrm{CI}=-0.010$ to $-0.002 ; p=0.002$; adjusted $p[8]$ $=0.008)$ and by $0.014 \mathrm{~mL}$ for every year of disease duration $(\beta$ $=-0.014 ; 95 \% \mathrm{CI}=-0.02$ to $-0.008 ; p=4 \times 10^{-6}$; adjusted $p$ $[8]=2 \times 10^{-5}$; figure 2 ).

The higher rate of $\mathrm{T} 2$ lesion volume accumulation $(\mathrm{mL})$ was associated with a higher $\mathrm{BVL}$ rate $(\beta=-0.15 ; 95 \% \mathrm{CI}=-0.17$ to -0.12 ; Cohen $\mathrm{f} 2=0.028 ; p<1 \times 10^{-10}$; adjusted $p[8]<1 \times$ $\left.10^{-10}\right)$, higher annualized change in EDSS $(\beta=0.02 ; 95 \%$ $\mathrm{CI}=0.01-0.03$; Cohen $\mathrm{f} 2=0.003 ; p=0.0002$; adjusted $p[8]=$ $0.0008)$, and higher relapse rate $(\beta=0.20 ; 95 \% \mathrm{CI}=0.05-0.34$; Cohen $\mathrm{f} 2=0.001 ; p=0.007$; adjusted $p[8]=0.017)$. The strength of the association between the rate of accumulation of T2 lesion volume and BVL rate was similar among patients of different ages and disease durations (interaction between age and T2 lesion volume accumulation rate: $\beta=0.0003$; $p=0.83$; adjusted $p[8]=0.83$; interaction between disease duration and $\mathrm{T} 2$ lesion volume accumulation rate: $\beta=0.004 ; p=0.027$; adjusted $p[8]=0.054)$.

\section{Tissue-Specific Brain Volume Analysis}

The sensitivity analysis that included the 348 trial patients ( 1,569 scans) from the ASA and the SET cohorts confirmed the absence of relationship between age and BVL rate $(\beta=$ $-0.001 ; p=0.75$; adjusted $p[8]=1.00)$. Similarly, the rate of segmented volume loss was constant across patient ages: white matter $(\beta=-0.011 ; p=0.014$; adjusted $p[8]=0.70)$, total gray matter $(\beta=-0.016 ; p=0.011$; adjusted $p[8]=$ $0.066)$, deep gray matter $(\beta=-0.019 ; p=0.046$; adjusted $p[8]=0.18)$, thalamic $(\beta=-0.017 ; p=0.10$; adjusted $p[8]=$ $0.30)$, and lateral ventricle volume changes $(\beta=0.015 ; p=$ 0.56 ; adjusted $p[8]=1.00$; in all Cohen $\mathrm{f} 2<0.005$ ). Only on 1 occasion, a weak association between age and cortical gray matter \% volume loss was seen $(\beta=-0.019 ; p=0.004$; Cohen $\mathrm{f} 2=0.006$; adjusted $p[8]=0.028)$. This association was 

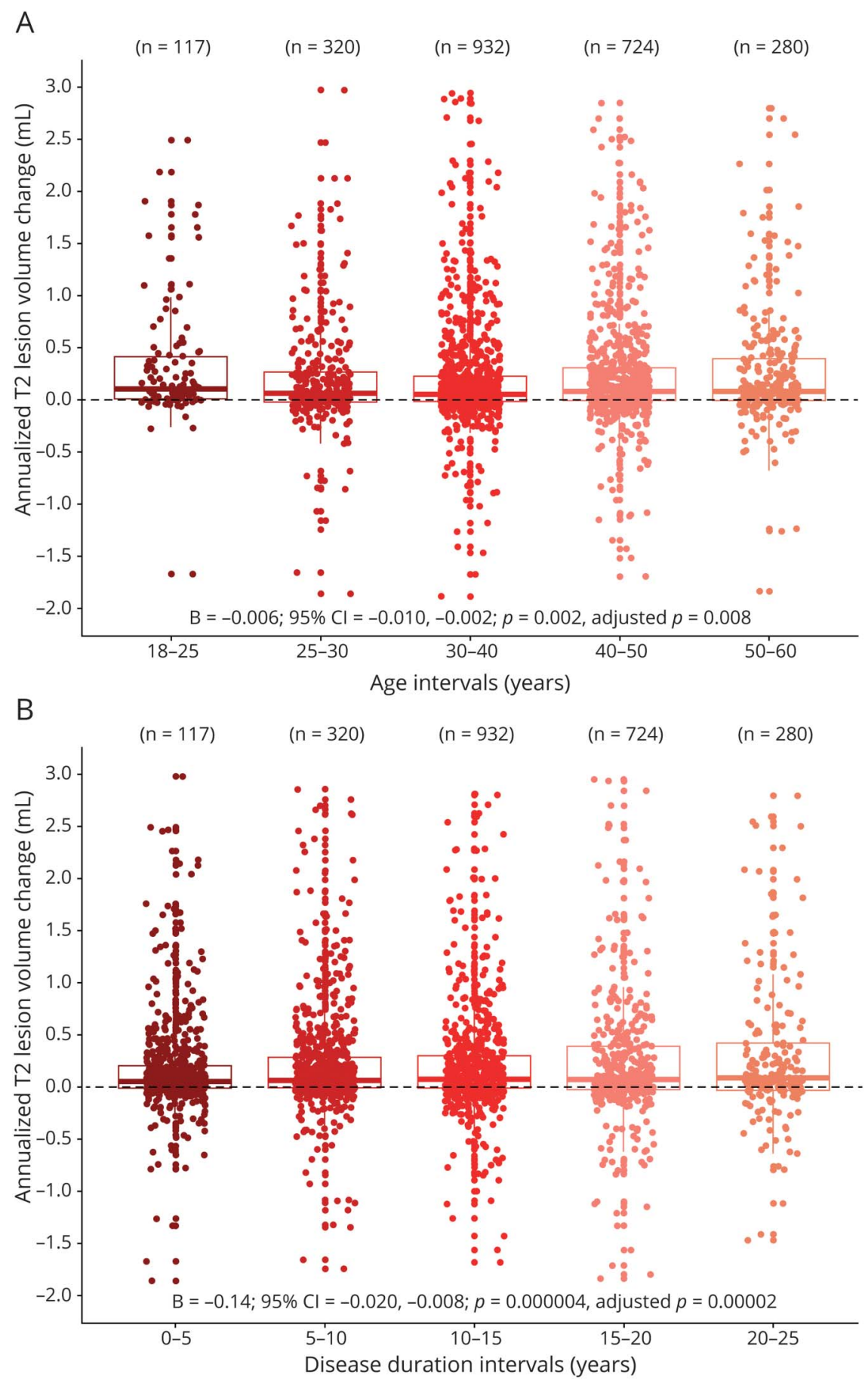

Mean annualized T2 lesion volume absolute changes in different age $(A)$ and disease duration (B) intervals. Statistical analysis was performed on continuous data.

confirmed in analysis of patients treated only with low-efficacy DMTs $(\beta=-0.021$; Cohen $\mathrm{f} 2=0.008 ; p=0.002$; adjusted $p$ $[8]=0.016)$.

The magnitude of the association between disease duration and the BVL rate was similar to the primary analysis, but did not reach the level of formal statistical significance due to smaller sample size $(\beta=-0.009 ; p=0.33$; adjusted $p[7]=$ $0.96)$. Similarly, we did not find any evidence for associations of disease duration with segmented brain volumes: white matter $(\beta=-0.016 ; p=0.32$; adjusted $p[7]=0.96)$, total gray matter $(\beta=-0.024 ; p=0.094$; adjusted $p[7]=0.38)$, cortical gray matter $(\beta=-0.028 ; p=0.045$; adjusted $p[7]=0.27)$, deep gray matter $(\beta=-0.014 ; p=0.51$; adjusted $p[7]=0.27)$, thalamic $(\beta=-0.013 ; p=0.023$; adjusted $p[7]=0.16)$, and lateral ventricle volume changes $(\beta=0.008 ; p=0.73$; adjusted $p[7]=0.96$; in all Cohen $\mathrm{f} 2<0.005$ ) (figure e-5, links.lww. com/NXI/A447). 


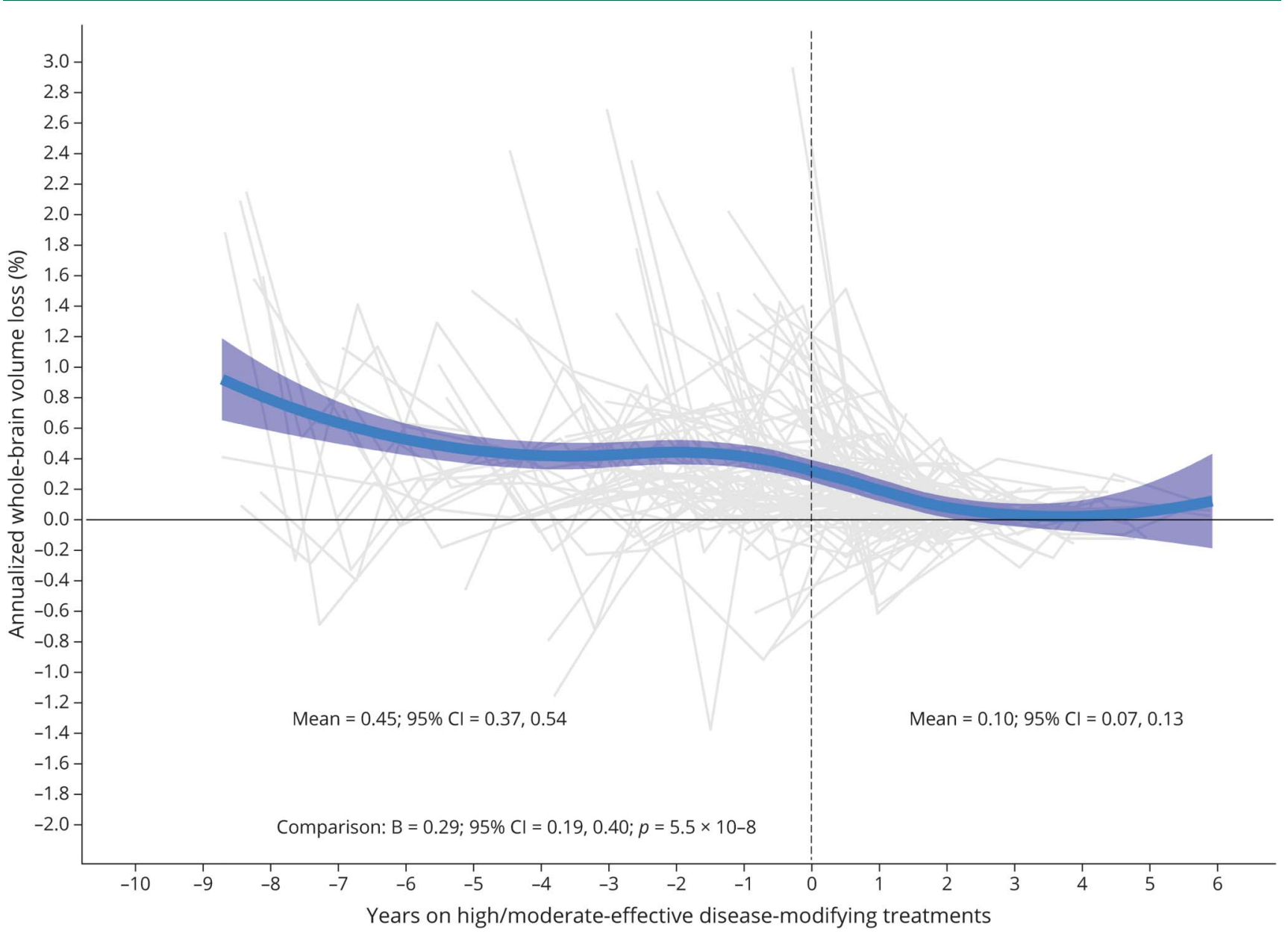

Evolution of annualized whole-brain volume loss after escalation from the low-efficacy on high/moderate-efficacy disease-modifying treatments. The treatment change occurred at time 0.

\section{An Effect of Disease-Modifying Treatment on Brain Volume Loss}

In a subgroup of 94 patients who escalated DMTs (609 MRI scans), the mean BVL rates substantially decreased following treatment escalation (before: $-0.45 ; 95 \% \mathrm{CI}=-0.54$ to -0.37 vs after: $-0.10 ; 95 \% \mathrm{CI}=-0.13$ to -0.07$)$. Differences in BVL rates following treatment escalation were confirmed in adjusted mixed models, where treatment escalation resulted in a mean reduction of the BVL rate by $0.29 \%(\beta=-0.29 ; 95 \% \mathrm{CI}=-0.40$ to -0.19 ; Cohen $\mathrm{f} 2=0.133 ; p=5.5 \times 10^{-8}$ ) (figure 3 ). An average time between the cessation of low-efficacy DMT and the initiation of high/moderate-efficacy DMT was 1.9 months (range 0-11.4 months).

\section{Discussion}

In this large longitudinal study of patients with RRMS, the rate of BVL declined with longer disease duration but not with older age. Patients with shorter disease duration showed marginally higher rates of BVL than patients with longer disease history; however, the effect of disease duration on the BVL rate was minimal. Similarly, higher rate of BVL was weakly associated with the higher frequency of recent relapses and greater disability, although the magnitude of these associations was relatively small. On the other hand, a stronger association was found between the rate of BVL and concurrent enlargement of cerebral T2 lesions or time to future confirmed disability worsening. Importantly, BVL was ameliorated by escalation of MS therapy. We did not find any clinically relevant associations between time and the rate of tissue-specific BVL.

In a study of 140 patients with MS, Andorra et al. ${ }^{8}$ showed that the rate of BVL is accelerated during the first 5 years after disease onset, followed by its subsequent stabilisation. Similarly, we have demonstrated that the rate of BVL is slightly higher in patients earlier after disease onset. Each year of disease duration was associated with a mean reduction in BVL rate of $0.013 \%$ (or $0.009 \%$ in patients treated exclusively with low-efficacy DMTs). However, the decline in the BVL rate was constant throughout the disease course.

Another large study of 963 untreated patients (1,926 time points) reported that the BVL rate was independent of MS 
subtype, age, or disease duration, when adjusting for baseline brain volume. ${ }^{6}$ A recent study of 1,417 patients (3,604 time points) showed accelerated cortical gray matter atrophy in SPMS compared with RRMS. ${ }^{2}$ These observations are complementary. First, in SPMS, the disease is more progressed and patients reaching secondary progressive phase have in general greater disease severity than age-matched patients with CIS or RRMS. Second, SPMS is usually associated with older age and longer disease history, and other determinants such as aging ${ }^{15}$ and comorbidities ${ }^{27}$ start to contribute to BVL.

The rates of BVL found in our study were comparable with those reported in previous studies that included treated MS patients, ${ }^{6,8,14,28}$ In the present study, we focused on relatively young cohorts of patients with short disease duration and with relatively more active disease from its onset. In keeping with a published study, ${ }^{13}$ our work showed that the frequency of relapses is associated with the rate of BVL; however, the magnitude of the association between recent relapse activity and the rate of BVL was marginal. The rate of recent increase in the cerebral T2 lesion burden was moderately associated with the rate of BVL. ${ }^{13,29,30}$ Of interest, the weak association between relapse frequency and the BVL rate was relatively stronger in younger patients but not specifically during early disease. These observations may suggest that the rate of BVL is closely related to the episodes of neuroinflammation that underlie formation of new or reactivation of existing cerebral lesions that presents clinically as MS relapses. This is supported by the demonstrated strong relationship between acute lesional inflammation and early axonal disruption as well as subsequent degeneration of chronically demyelinated axons resulting in brain tissue loss. ${ }^{3-34}$ In addition, we found a relationship between early BVL and future disability progression over long-term followup, which is in agreement with previous research and supports clinical relevance of BVL. ${ }^{1-5,9,13,17}$ As expected, we found a somewhat stronger relationship between BVL and disability worsening in older patients. ${ }^{35}$ Taken together, these observations may suggest that although formation of new lesions is a strong driver of BVL among younger patients, the loss of brain tissue in turn determines impaired neurologic function at older age. Given that the effect of age on the association between the rate of BVL and relapse frequency or disability worsening was only minimal, clinical relevance of these findings requires further validation.

Establishing the definition of BVL that is considered normal in the context of normal aging is a key step toward clinical interpretation of BVL in MS. The constant rate of BVL across different age groups of patients with MS simplifies the clinical use of this metric. However, the drivers of BVL may differ over time. In elderly patients, the aging process and comorbid diseases are likely to contribute more to the overall BVL than in younger patients, and therefore, a faster BVL in older patients may not always represent accelerated brain atrophy due specifically to MS. ${ }^{14,36}$ On the contrary, in early MS, higher BVL rates are more likely to indicate high level of disease activity requiring more aggressive therapy. Considering the relationship between early BVL and future development of disability, ${ }^{2,4,9,13}$ a neurologist should be particularly vigilant in screening for accelerated BVL in patients recently diagnosed with MS. This has become of particular importance as the new and emerging DMTs hold the promise of decelerating the rate of BVL and the loss of neurologic capacity. ${ }^{37,38}$ Our present study has also shown that escalation of DMT can help normalize the increased rate of BVL. ${ }^{28,39}$

\section{Limitations}

Our study included mostly patients treated with DMTs; therefore, the effect of pseudo-atrophy may be present. ${ }^{20,21}$ To minimize this, we analyzed only MRI scans performed at least 12 months after DMT initiation. Importantly, to minimize a potential effect of DMT escalation, we replicated our findings in a subgroup of patients who were treated exclusively with low-efficacy DMTs.

Moreover, the majority of patients started MRI monitoring at the time of disease onset or at presentation with relapses; therefore, the regression-to-the-mean phenomenon might play a role in detecting marginally increased rates of BVL in early disease stages. In addition, patients with RRMS who remained untreated over the long term are probably those with a favorable disease phenotype. On the other hand, untreated patients during early disease stages represent a more heterogeneous population.

It would be expected that the rate of BVL becomes accelerated in older age. ${ }^{2,9,14,36}$ In practice, older patients with more severe disability and during the transition to SPMS often receive less frequent MRI monitoring due to limited therapeutic options. Such patients might be slightly underrepresented in this study and explain the absence of accelerated rate of BVL in older patients.

Although BVL rates showed in the study are in agreement with previous research, ${ }^{6,8,14,28}$ the differences in volumetric outcomes are often driven by differences in MRI acquisition and volumetric technique. Therefore, absolute changes of BVL rates should not be directly compared with the results from other studies, in particular where different methodology was used.

In the available cohorts, almost $70 \%$ of time points were recorded during treatment with low-efficacy DMTs. Our results need to be replicated among patients treated with more contemporary, high/moderate-efficacy DMTs.

Finally, the study did not include patients with progressive MS, which may limit generalizability of our finding to relapsing MS only. We did not study healthy controls and therefore cannot comment on the contribution of age vs MS to the total observed BVL. ${ }^{14,36}$

In this longitudinal study of a large sample of patients with RRMS, we did not find a clinically relevant relationship between BVL rate and age or disease duration. Accelerated BVL was 
weakly associated with concurrent higher disease activity and eventually led to more profound neurologic disability. Evidence of higher rate of BVL should prompt the consideration of escalation of MS therapy to prevent neurologic disability.

\section{Acknowledgment}

The authors thank statistician Vaclav Capek, MS, $\mathrm{PhD}$, from the Department of Neurology and Center of Clinical Neuroscience, Charles University in Prague for help with developing a data analysis plan, review of statistics, and help with statistical analysis and interpretation. The authors also thank patients who participated in this study.

\section{Study Funding}

Study funded by Sanofi (GZ-2017-11718), the Czech Ministry of Education project Progress Q27/LF1, and Czech Ministry of Health project RVO-VFN64165 and NV18-04-00168.

\section{Disclosure}

The authors of the study have been consultants or received support for conference travel or research from pharmaceutical companies producing immune-modulatory drugs for escalation treatment in MS. T. Uher received financial support for conference travel and speaker honoraria from Biogen Idec, Novartis, Roche, Genzyme, and Merck Serono and support for research activities from Biogen Idec and Sanofi (GZ-201711718). J. Krasensky received financial support for conference travel and research activities from Biogen Idec. C.B. Malpas and N. Bergsland have nothing to disclose. M. Dwyer received personal compensation from Claret Medical and Keystone Heart Ltd. and research grant support from Novartis, Bristol Myers Squibb, and Mapi Pharma. E.K. Havrdova received speaker honoraria and consultant fees from Biogen Idec, Merck Serono, Novartis, Genzyme, Teva, Actelion, and Receptos and support for research activities from Biogen Idec and Merck Serono. M. Vaneckova received compensation for travel, speaker honoraria and consultant fees from Biogen Idec, Novartis, Merck Serono, Genzyme, Roche and Teva, and support for research activities from Biogen Idec. D. Horakova received compensation for travel, speaker honoraria and consultant fees from Biogen Idec, Novartis, Merck Serono, Bayer Shering, and Teva, and support for research activities from Biogen Idec. R. Zivadinov received personal compensation from EMD Serono, Sanofi, Novartis, BristolMyers Squibb, and Keystone Heart for speaking and consultant fees. He received financial support for research activities from Sanofi, Novartis, Bristol-Myers Squibb, Mapi Pharma, Protembis, V-VAWE Medical, and Keystone Heart. Dr. Zivadinov serves on the editorial board of $J$ Alzh Dis, BMC Med, BMC Neurol, Vein and Lymphatics, and Clinical CNS Drugs. Tomas Kalincik served on scientific advisory boards for Roche, Genzyme-Sanofi, Novartis, Merck, and Biogen, steering committee for Brain Atrophy Initiative by Genzyme, received conference travel support and/or speaker honoraria from WebMD Global, Novartis, Biogen, Genzyme-Sanofi, Teva, BioCSL, and Merck, and received research support from Biogen. Go to Neurology.org/NN for full disclosures.

\section{Publication History}

Received by Neurology: Neuroimmunology \& Neuroinflammation January 15, 2020. Accepted in final form January 5, 2021.

Appendix Authors

\begin{tabular}{|c|c|c|}
\hline Name & Location & Contribution \\
\hline $\begin{array}{l}\text { Tomas } \\
\text { Uher, MD, } \\
\text { PhD }\end{array}$ & $\begin{array}{l}\text { University of Melbourne, } \\
\text { VIC, Australia and Charles } \\
\text { University, Prague, Czech } \\
\text { Republic }\end{array}$ & $\begin{array}{l}\text { Study concept and design } \\
\text { and data analysis and } \\
\text { manuscript preparation }\end{array}$ \\
\hline $\begin{array}{l}\text { Jan } \\
\text { Krasensky, } \\
\text { MS }\end{array}$ & $\begin{array}{l}\text { Charles University, } \\
\text { Prague, Czech Republic }\end{array}$ & $\begin{array}{l}\text { MRI data analysis and data } \\
\text { interpretation }\end{array}$ \\
\hline $\begin{array}{l}\text { Charles B. } \\
\text { Malpas, } \\
\text { MPsych, } \\
\text { PhD }\end{array}$ & $\begin{array}{l}\text { University of Melbourne, } \\
\text { VIC, Australia }\end{array}$ & $\begin{array}{l}\text { Statistical analysis and } \\
\text { manuscript preparation }\end{array}$ \\
\hline $\begin{array}{l}\text { Niels } \\
\text { Bergsland, } \\
\text { MS, PhD }\end{array}$ & University at Buffalo, NY & $\begin{array}{l}\text { MRI data analysis, data } \\
\text { interpretation, and } \\
\text { manuscript preparation }\end{array}$ \\
\hline $\begin{array}{l}\text { Michael G. } \\
\text { Dwyer, MS, } \\
\text { PhD }\end{array}$ & University at Buffalo, NY & $\begin{array}{l}\text { MRI data analysis and data } \\
\text { interpretation }\end{array}$ \\
\hline $\begin{array}{l}\text { Eva Kubala } \\
\text { Havrdova, } \\
\text { MD, PhD }\end{array}$ & $\begin{array}{l}\text { Charles University, } \\
\text { Prague, Czech Republic }\end{array}$ & $\begin{array}{l}\text { Study concept and design } \\
\text { and data interpretation }\end{array}$ \\
\hline $\begin{array}{l}\text { Manuela } \\
\text { Vaneckova, } \\
\text { MD, PhD }\end{array}$ & $\begin{array}{l}\text { Charles University, } \\
\text { Prague, Czech Republic }\end{array}$ & $\begin{array}{l}\text { MRI data analysis and data } \\
\text { interpretation }\end{array}$ \\
\hline $\begin{array}{l}\text { Dana } \\
\text { Horakova, } \\
\text { MD, PhD }\end{array}$ & $\begin{array}{l}\text { Charles University, } \\
\text { Prague, Czech Republic }\end{array}$ & $\begin{array}{l}\text { Study concept and design } \\
\text { and data interpretation }\end{array}$ \\
\hline $\begin{array}{l}\text { Robert } \\
\text { Zivadinov, } \\
\text { MD, PhD }\end{array}$ & University at Buffalo, NY & $\begin{array}{l}\text { MRI data analysis and data } \\
\text { interpretation }\end{array}$ \\
\hline $\begin{array}{l}\text { Tomas } \\
\text { Kalincik, } \\
\text { MD, PhD }\end{array}$ & $\begin{array}{l}\text { University of Melbourne, } \\
\text { VIC, Australia }\end{array}$ & $\begin{array}{l}\text { Study concept and design, } \\
\text { data interpretation, and } \\
\text { manuscript preparation }\end{array}$ \\
\hline
\end{tabular}

\section{References}

1. Rocca MA, Battaglini M, Benedict RH, et al. Brain MRI atrophy quantification in MS: from methods to clinical application. Neurology 2017;88:403-413.

2. Eshaghi A, Prados F, Brownlee WJ, et al. Deep gray matter volume loss drives disability worsening in multiple sclerosis. Ann Neurol 2018;83:210-222.

3. Azevedo CJ, Pelletier D. Whole-brain atrophy: ready for implementation into clinical decision-making in multiple sclerosis? Curr Opin Neurol 2016;29:237-242.

4. Sastre-Garriga J, Pareto D, Battaglini M, et al. MAGNIMS consensus recommendations on the use of brain and spinal cord atrophy measures in clinical practice. Nat Rev Neurol 2020;16:171-182.

5. Filippi M, Rocca MA, Barkhof F, et al. Association between pathological and MRI findings in multiple sclerosis. Lancet Neurol 2012;11:349-360.

6. De Stefano N, Giorgio A, Battaglini M, et al. Assessing brain atrophy rates in a large population of untreated multiple sclerosis subtypes. Neurology 2010;74:1868-1876.

7. Kalkers NF, Ameziane N, Bot JC, Minneboo A, Polman CH, Barkhof F. Longitudinal brain volume measurement in multiple sclerosis: rate of brain atrophy is independent of the disease subtype. Arch Neurol 2002;59:1572-1576.

8. Andorra M, Nakamura K, Lampert EJ, et al. Assessing biological and methodological aspects of brain volume loss in multiple sclerosis. JAMA Neurol 2018;75:1246-1255.

9. Rudick RA, Lee JC, Nakamura K, Fisher E. Gray matter atrophy correlates with MS disability progression measured with MSFC but not EDSS. J Neurol Sci 2009;282: 106-111.

10. Scalfari A, Lederer C, Daumer M, Nicholas R, Ebers GC, Muraro PA. The relationship of age with the clinical phenotype in multiple sclerosis. Mult Scler 2016;22: $1750-1758$.

11. Kalincik T, Vivek V, Jokubaitis V, et al. Sex as a determinant of relapse incidence and progressive course of multiple sclerosis. Brain 2013;136:3609-3617. 
12. Held U, Heigenhauser L, Shang C, Kappos L, Polman C; Sylvia Lawry Centre for MSR. Predictors of relapse rate in MS clinical trials. Neurology 2005;65:1769-1773.

13. Radue EW, Barkhof F, Kappos L, et al. Correlation between brain volume loss and clinical and MRI outcomes in multiple sclerosis. Neurology 2015;84:784-793.

14. Azevedo CJ, Cen SY, Jaberzadeh A, Zheng L, Hauser SL, Pelletier D. Contribution of normal aging to brain atrophy in MS. Neurol Neuroimmunol Neuroinflamm 2019;6: e616. doi: 10.1212/NXI.0000000000000616.

15. Hedman AM, van Haren NE, Schnack HG, Kahn RS, Hulshoff Pol HE. Human brain changes across the life span: a review of 56 longitudinal magnetic resonance imaging studies. Hum Brain Mapp 2012;33:1987-2002.

16. Havrdova E, Zivadinov R, Krasensky J, et al. Randomized study of interferon beta-1a, low-dose azathioprine, and low-dose corticosteroids in multiple sclerosis. Mult Scler 2009; 15:965-976

17. Kalincik T, Vaneckova M, Tyblova M, et al. Volumetric MRI markers and predictors of disease activity in early multiple sclerosis: a longitudinal cohort study. PLoS One 2012;7:e50101.

18. Uher T, Vaneckova M, Krasensky J, et al. Pathological cut-offs of global and regional brain volume loss in multiple sclerosis. Mult Scler 2019;25:541-553.

19. Kalincik T, Cutter G, Spelman T, et al. Defining reliable disability outcomes in multiple sclerosis. Brain 2015;138:3287-3298.

20. Zivadinov R, Reder AT, Filippi M, et al. Mechanisms of action of disease-modifying agents and brain volume changes in multiple sclerosis. Neurology 2008;71:136-144.

21. Zivadinov R, Rudick RA, De Masi R, et al. Effects of IV methylprednisolone on brain atrophy in relapsing-remitting MS. Neurology 2001;57:1239-1247.

22. Gelineau-Morel R, Tomassini V, Jenkinson M, Johansen-Berg H, Matthews PM, Palace J. The effect of hypointense white matter lesions on automated gray matter segmentation in multiple sclerosis. Hum Brain Mapp 2012;33:2802-2814.

23. Smith SM, Zhang $\mathrm{Y}$, Jenkinson $\mathrm{M}$, et al. Accurate, robust, and automated longitudinal and cross-sectional brain change analysis. Neuroimage 2002;17:479-489.

24. Patenaude B, Smith SM, Kennedy DN, Jenkinson M. A Bayesian model of shape and appearance for subcortical brain segmentation. Neuroimage 2011;56:907-922.

25. Dwyer MG, Bergsland N, Zivadinov R. Improved longitudinal gray and white matter atrophy assessment via application of a 4-dimensional hidden Markov random field model. Neuroimage 2014;90:207-217.
26. Selya AS, Rose JS, Dierker LC, Hedeker D, Mermelstein RJ. A practical guide to calculating Cohen's $\mathrm{f}(2)$, a measure of local effect size, from PROC MIXED. Front Psychol 2012;3:111.

27. Jakimovski D, Gandhi S, Paunkoski I, et al. Hypertension and heart disease are associated with development of brain atrophy in multiple sclerosis: a 5-year longitudinal study. Eur J Neurol 2019;26:87-e88.

28. Arnold DL, Fisher E, Brinar VV, et al. Superior MRI outcomes with alemtuzumab compared with subcutaneous interferon beta-1a in MS. Neurology 2016;87:1464-1472.

29. Varosanec M, Uher T, Horakova D, et al. Longitudinal mixed-effect model analysis of the association between global and tissue-specific brain atrophy and lesion accumulation in patients with clinically isolated syndrome. AJNR Am J Neuroradiol 2015;36: 1457-1464.

30. Chard DT, Brex PA, Ciccarelli O, et al. The longitudinal relation between brain lesion load and atrophy in multiple sclerosis: a 14 year follow up study. J Neurol Neurosurg Psychiatry 2003;74:1551-1554.

31. Frischer JM, Weigand SD, Guo Y, et al. Clinical and pathological insights into the dynamic nature of the white matter multiple sclerosis plaque. Ann Neurol 2015;78:710-721.

32. Trapp BD, Peterson J, Ransohoff RM, Rudick R, Mork S, Bo L. Axonal transection in the lesions of multiple sclerosis. N Engl J Med 1998;338:278-285.

33. Frischer JM, Bramow S, Dal-Bianco A, et al. The relation between inflammation and neurodegeneration in multiple sclerosis brains. Brain 2009;132:1175-1189.

34. Trapp BD, Nave KA. Multiple sclerosis: an immune or neurodegenerative disorder? Annu Rev Neurosci 2008;31:247-269.

35. Trojano M, Liguori M, Bosco Zimatore G, et al. Age-related disability in multiple sclerosis. Ann Neurol 2002;51:475-480.

36. Battaglini M, Gentile G, Luchetti L, et al. Lifespan normative data on rates of brain volume changes. Neurobiol Aging 2019;81:30-37.

37. He A, Merkel B, Brown JWL, et al. Timing of high-efficacy therapy for multiple sclerosis: a retrospective observational cohort study. Lancet Neurol 2020;19:307-316.

38. Brown JWL, Coles A, Horakova D, et al. Association of initial disease-modifying therapy with later conversion to secondary progressive multiple sclerosis. JAMA 2019; 321:175-187.

39. Gaetano L, Haring DA, Radue EW, et al. Fingolimod effect on gray matter, thalamus, and white matter in patients with multiple sclerosis. Neurology 2018;90:e1324-e1332. 


\section{Neurology \\ Neuroimmunology \& Neuroinflammation}

Evolution of Brain Volume Loss Rates in Early Stages of Multiple Sclerosis

Tomas Uher, Jan Krasensky, Charles Malpas, et al.

Neurol Neuroimmunol Neuroinflamm 2021;8;

DOI 10.1212/NXI.0000000000000979

This information is current as of March 16, 2021

\section{Updated Information \& Services}

References

Subspecialty Collections

Permissions \& Licensing

\section{Reprints}

including high resolution figures, can be found at:

http://nn.neurology.org/content/8/3/e979.full.html

This article cites 39 articles, 3 of which you can access for free at: http://nn.neurology.org/content/8/3/e979.full.html\#\#ref-list-1

This article, along with others on similar topics, appears in the following collection(s):

Multiple sclerosis

http://nn.neurology.org//cgi/collection/multiple_sclerosis

Volumetric MRI

http://nn.neurology.org//cgi/collection/volumetric_mri

Information about reproducing this article in parts (figures,tables) or in its entirety can be found online at:

http://nn.neurology.org/misc/about.xhtml\#permissions

Information about ordering reprints can be found online:

http://nn.neurology.org/misc/addir.xhtml\#reprintsus

Neurol Neuroimmunol Neuroinflamm is an official journal of the American Academy of Neurology.

Published since April 2014, it is an open-access, online-only, continuous publication journal. Copyright

Copyright (C) 2021 The Author(s). Published by Wolters Kluwer Health, Inc. on behalf of the American

Academy of Neurology.. All rights reserved. Online ISSN: 2332-7812.

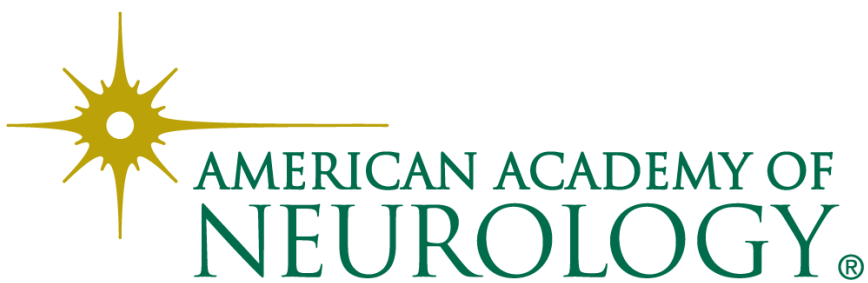

